\title{
An Energy-Efficient and Low-Latency MAC Protocol in Smart Grid (SG-MAC)
}

\author{
Ehsan Banari, Sayyed Majid Mazinani, Member, IACSIT, and Iman Sariri Ajili
}

\begin{abstract}
In this paper we propose a new protocol -namedSGMAC for reducing the energy consumption and shortening the time of data sending in smart grid to control media Access. This protocol uses sleeping and waking intervals distinct in the same duty cycle and the sender node will participate only in the period of receiving node over the ownership of the channel.

Furthermore, we have compared the proposed protocol from the point of view of energy and delay with IEEE802.11 and SMAC in two different protocol topologies. In the simulation results illustrated that SG-MAC protocol using data gathering tree and apply collection sleep and waking intervals, less delay in comparison with the SMAC protocol and much less energy consumption in comparison with the IEEE802.11.
\end{abstract}

Index Terms-Smart grid (SG), medium channel access control (MAC), sensor-MAC (S-MAC), request to send (RTS), clear to send (CTS).

\section{INTRODUCTION AND RELATED WORK}

The smart grid [1], [2] is an upcoming technology that can control the power load via price signaling [3]. By using protocols and standards, Samples the environmental information needed by the source node and send it to the sink node [4].

By using two-way communication, the latest technology and communication, improve Quality of service, efficiency and reliability [1].

The modernization of the electricity grid that can accommodate future demand growth is underway [5].

The protocols used in the smart grid to all nodes in the network bandwidth as well to allocate and have a little delay and reduce energy consumption as much as possible [6], [7].

Data traffic might be low for long periods of time, and it might exist high and complex traffic for short periods of time. Carrier sensing in smart grid, this can be done by listening to the network that this is causing a lot of energy, so it should be brief periods of listening. To reduce the risk of collision can be used as a back off mechanism [8], [9].

In IEEE 802.11 standard protocol, nodes listen to the channel to receive traffic. This protocol due to its simplicity and its power in solving a hidden terminal problem [10], [11] is widely used in smart grids. However, the energy consumption of IEEE 802.11 when nodes are in idle mode, is very high and, The main reason is listening in idle mode. In

Manuscript received April 25, 2014; revised July 1, 2014.

Ehsan Banari and Sayed Majid Mazinani are with Imam Reza International University (e-mail: ehsanbanari@yahoo.com, smajid_mazinani@yahoo.com).

Iman Sariri Ajili is with Iranian Construction Engineering Organization Province of Khorasan Razavi (e-mail: iman.sariri@yahoo.com).
IEEE 802.11, each node listens to all transmission from its neighbors to fully implement virtual listening that this is wasting a lot of energy [12], [13]. The measurements show that listening in idle mode is wasting more than 50 percent of the energy needed to get the data traffic [14].

SMAC protocol is an optimized protocol in energy consumption [15], [16] for reducing power consumption in half the timescales for competition mode and it operates for listening to an idle channel, a mechanism for periodic listen and sleep cycle at every node.

Updating the schedule, begins by sending a SYNC packet. The SMAC turns off the radio, during the sending of other nodes. If any node outside the unwanted transceiver is listening, cannot be aware of a continuous data, so it goes to sleep mode until the next cycle. Therefore in SMAC the process of data leading conductivity disrupt for each node that next jump into the sink outside the listening limitation, and delay will be longer. The IEEE 802.11 waste due to listening in idle mode makes high energy consumption.

The purpose of this paper is to represent a smart grid with minimal delay and low energy to achieve quality of service.

\section{SG-MAC PROTOCOL DESIGN}

In our proposed protocol, sender node only takes part in the period of awakening related to the receiving node in the competition over access to the medium and sending data. This caused reduction the possibility of collisions and additional listening problem will be greatly resolved.

In the proposed protocol, all off smart nodes will be in active mode in the synchronization time, and attend a competition for distribution of sync packets. A node that won in this competition will send a SYNC packet to its neighbor's nodes and make them aware of listen/sleep schedules of this node. Each smart node maintains a table that holds neighbor's synchronization and timing information is sleeping and waking neighbors. When sender node saw the awakening period of receiver node, Sending RTS and receiving CTS to compete for sending data begins. If a node fails in this competition, it must wait until the next frame.

As shown in Fig. 1, the frame structure in proposed protocol consists of two parts: a synchronization cycle that the synchronization signal is sent during this period and the sleep-wake cycle. Every sleep-wake cycle distributed into several pieces that the pieces called "sleep and wake period".

Each node has a period of time to send and receive data in a timeframe that can be awakened in the period and after the awakening period until the next frame comes next awakening period falls asleep except for a situation that it has a packet for sending. 


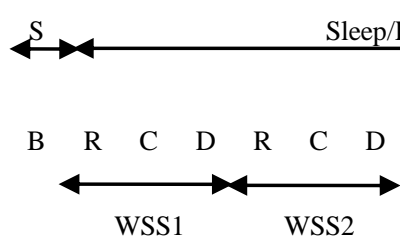

$\mathrm{S}$ : synchronization cycle

B: synchronization signal

WSS: waking interval

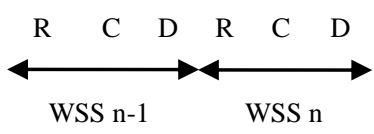

R: send/receive RTS interval

C: send/receive CTS interval

D: send/receive DATA interval

Fig. 1. Structure of the proposed protocol frame.

In this protocol to reduce energy consumption and latency and lag problems data forwarding interruption over multiple jump paths, we use a scheduler to wake up the nodes. As shown in Fig. 2 multiple sources of data delivered to a sink, makes a data gathering tree collection forms Unidirectional data flow direction in the sink and all nodes except the sink, that receive packets, To the next node in the data gathering tree sends data and all nodes are aware of the location of its neighbor nodes range awakening. In this protocol, location of the waking period depends on the each ID node.

We have reduced expects of receiver node by integrating data gathering tree and allocation ranges sleep nodes of transmitting to begin receiving period node. In this method we have assigned intervals to nodes by using a node-level in data gathering tree. The level of one node shows its distance to the root.

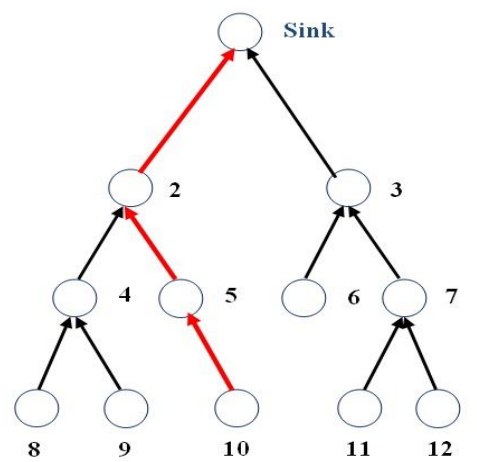

Fig. 2. Shows an example of a data gathering tree that marked the node.

Allocation ranges waking nodes using equation (1) is performed:

$$
S i=M-(i \bmod M)
$$

In relation (1), $i$ is the level number of nodes in data gathering tree, $S i$ shows the number of interval assigned to certain nodes and $M$ is the total number of intervals of sleep and waking in a frame. Advantage of this method is that if a node has data to send, waiting for the arrival probability of awakening the receiving node has a low range.

In Fig. 2, if node No.10 has a packet to send to node No. 1, according to (1) which also shown in Fig. 3, node No. 10 begins to send data to node No. 5 in second interval and node No. 5 send data to node No. 2 in third interval, finally node No. 2 apply to send to node No.1 which named sink in fourth interval. So in this example, the Data transmission from source to sink in a frame is performed.

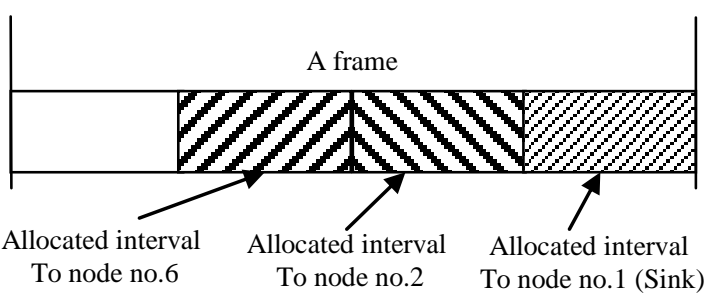

Fig. 3. Sending the packets from node 10 to node 1 in proposed protocol.

Numbers of sleep and waking intervals in each frame depends on performance of network application in use. In high-density networks require more sleep and wake intervals. Devoting the awaking range on the base of the node level is done in the data gathering tree.

As shown in Fig. 4; proposed algorithm is like that at first will broadcast synchronization frame. If every node does not have any data for sending it will go to Back off mode. Then supposed node will send RTS message to the sink node and the sink node will send CTS message to sender node which shows media is free. Finally data will be transmitted.

The algorithm proposed protocol is as follows:

\begin{tabular}{|c|c|}
\hline- & Nodes go to sleep \\
\hline- & IF (sync-period arrives) THEN \\
\hline- & Begin \\
\hline- & Nodes start Back-off \\
\hline- & IF (node (i) wins contention) THEN \\
\hline- & Send SYNC; \\
\hline- & End; \\
\hline- & IF (slot assigned to node (i)) THEN \\
\hline- & Begin \\
\hline- & Starts listening; \\
\hline- & IF (receive RTS) THEN \\
\hline- & Begin \\
\hline- & Transmit CTS; \\
\hline- & Start receiving DATA; \\
\hline- & Wait for next slot; \\
\hline- & Start Back-off; \\
\hline- & Transmit RTS; \\
\hline- & IF (receive CTS) THEN \\
\hline- & Start transmitting DATA; \\
\hline- & End; \\
\hline- & End; \\
\hline
\end{tabular}

Fig. 4. Algorithm of proposed protocol.

Our proposed protocol that explained the above steps simulation with NS-2 software and plot the graphs with MATLAB to have the results below:

Simulation parameters are shown in Table I.

TABLE I: RADIO PARAMETERS

\begin{tabular}{|l|l|}
\hline Radio Bandwidth & $100 \mathrm{kbps}$ \\
\hline Radio Transmission Range & $250 \mathrm{~m}$ \\
\hline Radio Interference Range & $550 \mathrm{~m}$ \\
\hline Data Packet Length & 100 Bytes \\
\hline Transmit Power & $0.66 \mathrm{~W}$ \\
\hline Receive Power & $0.395 \mathrm{~W}$ \\
\hline Idle Power & $0.35 \mathrm{~W}$ \\
\hline Sleep Power & $0 \mathrm{~W}$ \\
\hline Antenna height above the ground & $1.5 \mathrm{~m}$ \\
\hline
\end{tabular}




\section{CAlculation}

The first simulation is done on a chain with 7 step smart nodes. In this simulation the distance between any two adjacent smart nodes is 200 meters and each node has 250 meters strength.

Fig. 5 shows average packet delay by the nodes based on the number of steps and Fig. 6 shows the average delay of packets in various traffic conditions in three different protocols.

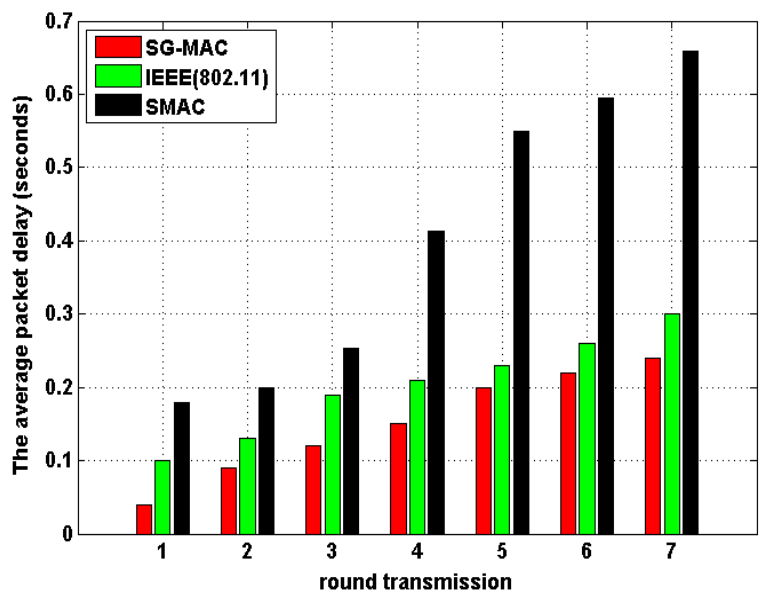

Fig. 5. average packet delay based on the number of steps.

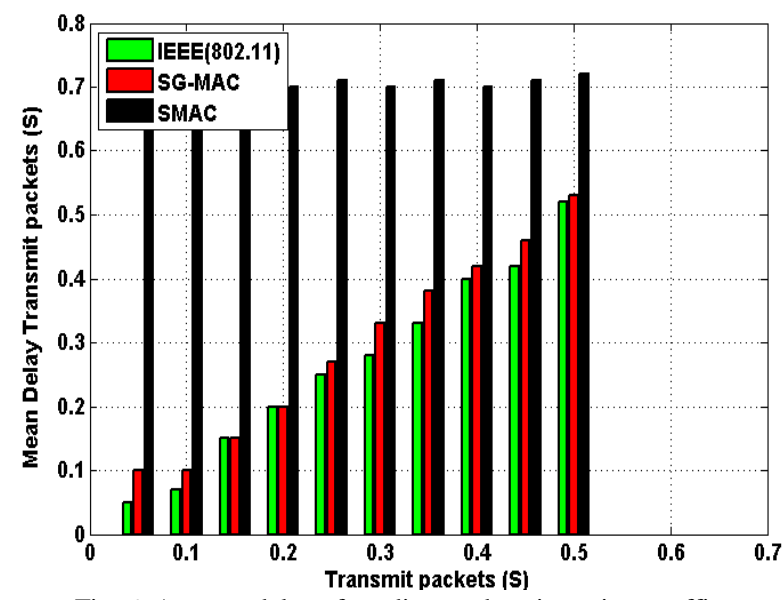

Fig. 6. Average delay of sending packets in various traffic.

As shown in Fig. 5 and Fig. 6 due to nodes being awake permanently, and lack of sleep in the IEEE 802.11 protocol, the protocol, has a good delay. Because SMAC has the longer sleep-wake intervals thus have more sleep delay before sending data to an intermediate node has to wait to get up the receiver. Because of using data gathering tree of SG-MAC protocol, this protocol, has less latency than SMAC.

Fig. 6 also due to the heavy traffic, the delay difference between the protocols is even more sensible than the light traffic.

In SMAC because of periodical sleep, sender should wait till receiver node wake up.

Fig. 7 shows total amount of energy consumption in terms of the number of steps and Fig. 8 shows total energy consumption in various traffic conditions.

As specified, in the IEEE 802.11 protocol, due to persistent active nodes and extra listening time being idle channel, nodes have to waste a lot of energy. In Both SMAC and
SG-MAC protocol, energy are almost equal and this is why these two protocols use the periods of sleep and waking.

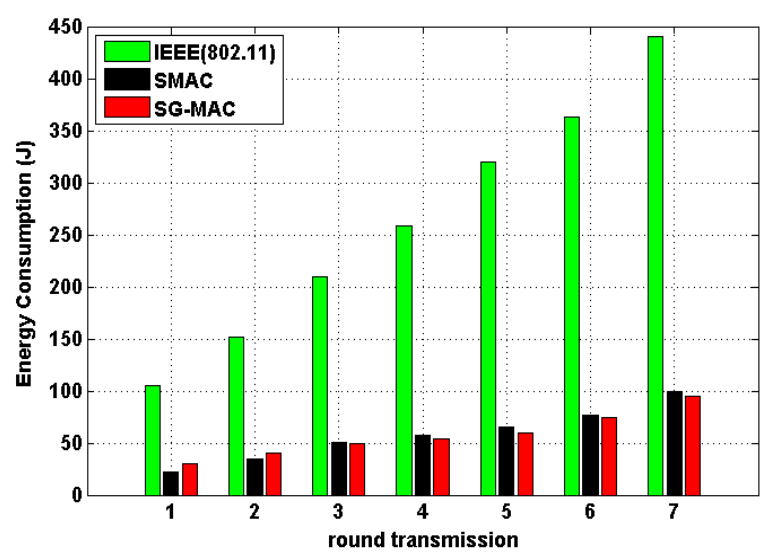

Fig. 7. Total energy consumption based on the number of steps.

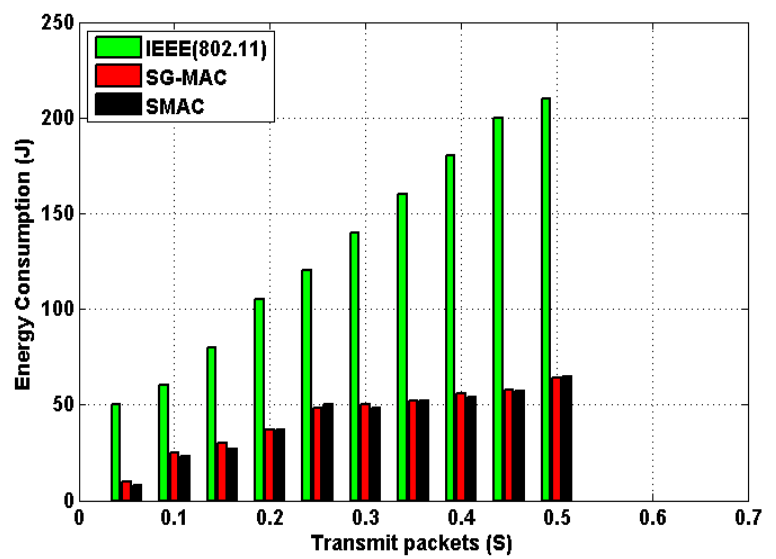

Fig. 8. Total energy consumption for packets in various traffic conditions.

The second simulation is generated based on a random data gathering tree. In this topology, 50 smart nodes are distributed randomly in a $500 \mathrm{~m} \times 1000 \mathrm{~m}$ area. Each source node has to send 10 packets. Numbers of sleep and waking intervals are considered as 4 .

Fig. 9 shows total delay of transmit packets and Fig. 10 shows total consumed energy in various traffic conditions by 50 smart nodes that has been calculated in this topology.

According to Fig. 9 and Fig. 10, in IEEE802.11, each node immediately starts the listening to the channel and then tries to send the packet to the next step while in the other two protocols, when a sender receives a packet to send to the next step must wait for the receiver to wake up. Intervals of sleep and waking in SG-MAC is shorter than SMAC. In SG-MAC protocol, usually when a node received a packet may immediately to send the packet in next interval to the receiver because the receiver is awake in this interval can receive the packet. Due to the limited number of awakening nodes in each interval, the probability of collision in proposed protocol, is less than SMAC protocol.

We have compared the performances both topologies from the viewpoint of energy and delay of the proposed protocol with two other protocols. We define a parameter named Energy $\times$ Latency.

The less energy consumption of nodes and the latency of packets in a protocol, the better efficient than the protocol, from the point of view of these two parameters. 


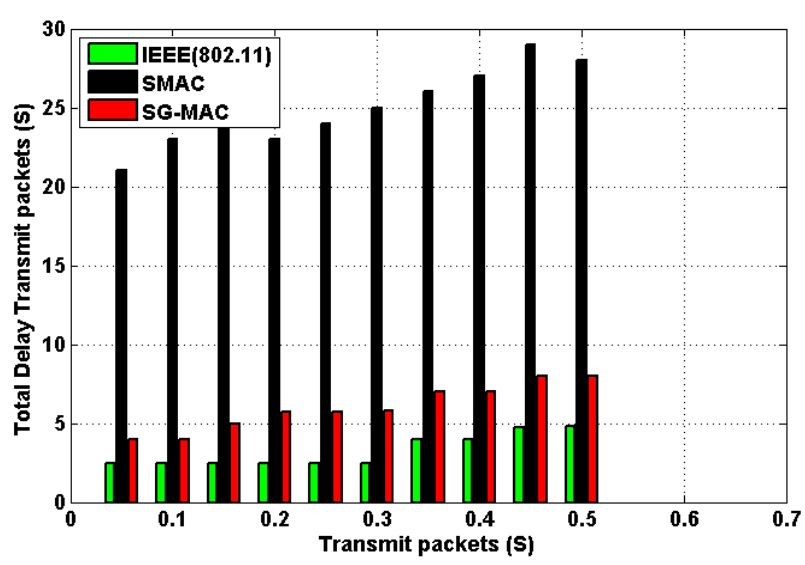

Fig. 9. The total delay of transmit packets during grid.

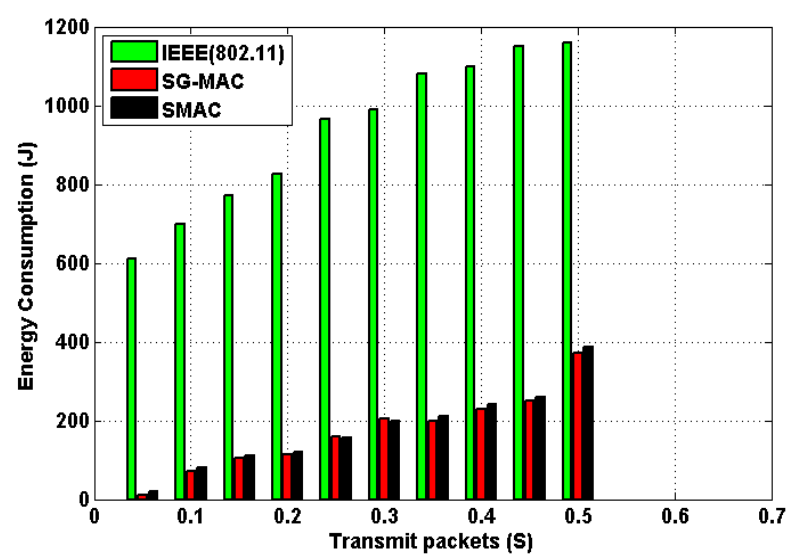

Fig. 10. The total energy consumption in various traffic conditions.

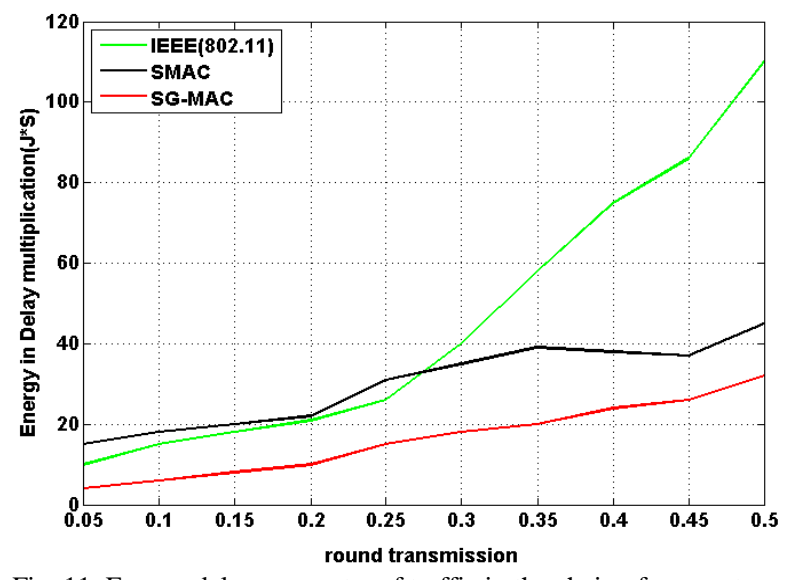

Fig. 11. Energy-delay parameter of traffic in the chain of seven steps. (topology I).

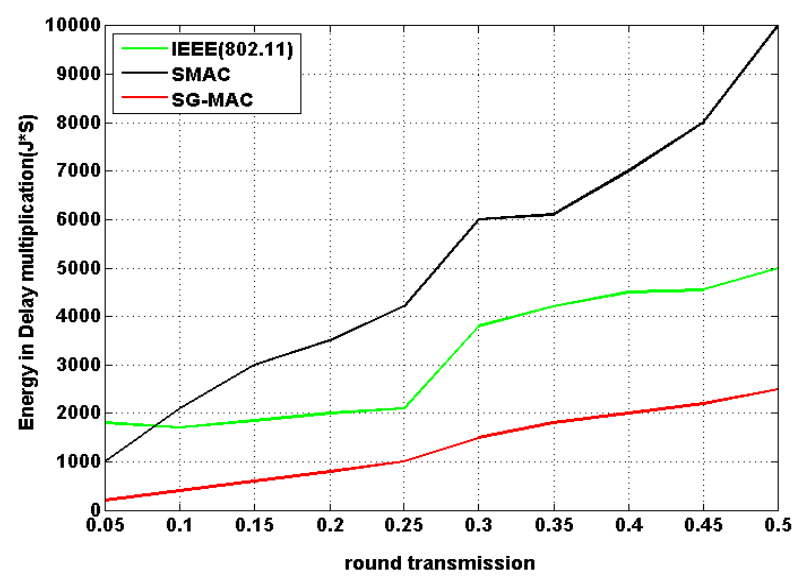

Fig. 12. Energy-delay parameter of traffic data collected accidental from the tree (topology II).
As in Fig. 11 and Fig. 12 are shown, the proposed SG-MAC protocol, shown in red color in comparison with the two other protocols in both topologies, in terms of the latency and energy has better performance that this shows the improvement of efficiency. This is why SG-MAC has less latency than SMAC and much less consumed energy than IEEE802.11.

\section{CONCLUSION}

In this paper we propose a new protocol, named SG-MAC for Medium Access Control. In this protocol, in each cycle, sleep and waking intervals were used separately and sender node only takes part in the period of awakening related to the receiving node in the competition over access to the medium and sending data. We have assigned intervals to each nodes by using a node-level in data gathering tree.

In the proposed protocol, nodes with different intake less than SMAC collision periods, the proposed protocol since the nodes have received at different times makes collisions less than SMAC. On the other hand, if the nodes in SMAC has the same duty cycle, they may coincide with each other in a competition over the ownership of a channel. Nodes can choose the same time Back off as the same is caused by repeatedly Collisions.

In addition, SG-MAC protocol, when a node received data in the frame immediately following the interval to send packets to the receiver. The simulation results indicate that SG-MAC is compared to SMAC much less delay In comparison with the IEEE802.11 because of using the intervals of sleep and waking has less consumed energy. The SG-MAC protocol from the point of view of these two parameters has better efficiency.

\section{REFERENCES}

[1] C. Cecati, G. Mokryani, A. Piccolo, and P. Siano, "An overview on the smart grid concept," in Proc. 36th Annual Conference on IEEE Industrial Electronics Society, 2010.

[2] N. Saputro, K. Akkaya, and S. Uludag, "A survey of routing protocols for smart grid communications," Computer Networks Elsevier, 2012.

[3] H. Li and W. Zhang, "QoS routing in smart grid," in IEEE Global Telecommunications Conf. GLOBECOM, 2010, pp. 1-6.

[4] Q. Wang, J. Wang, Y. Lin, J. Tang, and Z. Zhu, "Interference management for smart grid communication under cognitive wireless network," in Proc. Smart Grid Communications (SmartGridComm) 2012 IEEE Third International Conference, 2012, pp. 246-251.

[5] NIST, "NIST framework and roadmap for smart grid interoperability standards, release 1.0," 2010.

[6] I. S. Al. Anbagi, H. T. Mouftah, and M. Erol-Kantarci, "Design of a delay-sensitive WSN for wind generation monitoring in the smart grid," in Proc. Electrical and Computer Engineering (CCECE) 2011 24th Canadian Conference, 2011, pp. 1370-1373.

[7] G. J. Pottie and W. J. Kaiser, "Wireless integrated network sensor," Communications of the ACM, vol. 43, no. 5, pp. 51-58, May 2000.

[8] K. Oh, S. Woo, S. Sung, and K. Kim, "Improved energy efficiency of dmac with periodic full sleep cycle for wireless sensor networks with heavy traffics," in Proc. Intelligent Sensors، Sensor Networks and Information 2007, ISSNIP 3rd International Conference, 2007, pp. 85-89.

[9] G. Wolny, "Modified DMAC clustering algorithm for VANETs," in Proc. Systems and Networks Communications 2008, ICSNC 3rd International Conference, 2008, pp. 268-273.

[10] F. Tobagi and L. Kleinrock, "Packet switching in radio channels: Part II-the hidden terminal problem in carrier sense multiple access and the busy-tone solution," IEEE Transactions on Communications, vol. 23, no. 12 , pp. 1417-1433, Dec. 1975. 
[11] V. Bharghavan, A. Demers, S. Shenker, and L. Zhang, "Macaw: A media access protocol for wireless lans," in Proc. the ACM SIGCOMM Conference, 1994.

[12] Wireless LAN Medium Access Control (MAC) and Physical Layer (PHY) Specification, IEEE Standard 802.11-1997 Ed., 1997.

[13] Wireless LAN Medium Access Control (MAC) and Physical Layer (LHY) Specifications, IEEE Standard 802.11, 1999.

[14] W. Ye and J. Heidemann, "Medium access control in wireless sensor networks," Technical Reports ISI-TR-580, USC/Information Sciences Institute, Oct. 2003, pp. 1-8.

[15] W. Ye, J. Heidemann, and D. Estrin, "An energy-efficient MAC protocol for wireless sensor networks," in Proc. IEEE INFOCOM 2002, New York, NY , June 2002, pp. 1567-1576.

[16] W. Ye, J. Heidemann, and D. Estrin, "Medium access control with coordinated adaptive sleeping for wireless sensor networks," Networking IEEE/ACM Trans, vol. 12, no. 3, pp. 493-506, 2004.

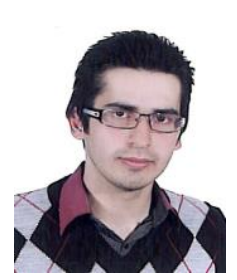

Ehsan Banari was born in Babol, Mazandaran, Iran on August 28, 1984. He received the B.S. degree in power electrical engineering from Babol Noshirvani University of Technology, Mazandaran, Iran in 2008 Currently he is a M.A. student in Imam Reza International University. His research interests include, quality of service, wireless sensor network, smart grid and cryptography (AES).

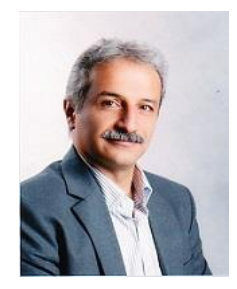

Sayyed Majid Mazinani was born in Mashhad, Iran on January 28, 1971. He received his bachelor degree in electronics from Ferdowsi University, Mashhad, Iran in 1994 and his master degree in remote sensing and image processing from Tarbiat Modarres University, Tehran, Iran in 1997. He worked in IRIB from 1999 to 2004. He also received his $\mathrm{PhD}$ in wireless sensor networks from Ferdowsi University, Mashhad, Iran in 2009. He is currently an assistant professor at the Faculty of Engineering in Imam Reza International University, Mashhad, Iran. He was the head of Department of Electrical and Computer Engineering from 2009 to 2012. His research interests include computer networks, wireless sensor networks and smart grids.

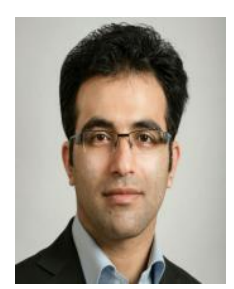

Iman Sariri Ajili received the B.S. degree in electrical engineering from Azad University, Gonabad, Iran, in 2004 and the M.S. degree in control engineering from Azad University, Gonabad, Iran, in 2008. From 2008 to 2009, he was a research assistant with the Institute of Data Mining, Sanaye Behshahr, Tehran, Iran. His research interest includes the development of data mining in smart grids, decision making with advanced data-mining in building 